\title{
Physical activity in pregnancy and postpartum depressive symptoms ina multiethnic cohort
}

Nilam Shakeel ${ }^{1}$, Kåre Rønn Richardsen ${ }^{2}$, Egil W. Martinsen ${ }^{3}$ Malin Eberhard-Gran 4,5,6 Kari Slinning $^{7}$, Anne Karen Jenum ${ }^{8}$

Introduction: There is strong evidencethat postpartum depression is associated with adverse health effects in the mother and infant. Fewstudies have exploredassociations between physical activity in pregnancy and postpartum depression. We aimed to investigate whether physical activity during pregnancy was inversely associated with postpartum depressive symptoms, PPDS in a multiethnic sample.

Method: Population-based, prospective cohort of 643 pregnant women (58 \% ethnic minorities) attending primary antenatal care from early pregnancy to postpartum in Oslo between 2008 and 2010. Data on demographics and health outcomes were collected during standardized interviews. PPDS was defined by a sum score $\geq 10$ from the Edinburgh Postnatal Depression Scale(EPDS), 3 months after birth. Physical activity was recorded with Sense Wear ${ }^{\text {TM }}$ Pro3 Armband (SWA) in gestational week 28 and defined as moderate-tovigorous intensity physical activity (MVPA) accumulated in bouts $\geq 10$ minutes.

Results: Women who accumulated $\geq 150$ MVPA minutes/week had significantly lower risk (OR=0.2, 95\%Cl: $0.06-0.90)$, for PPDS compared to those who did not accumulate any minutes/week of MVPA, adjusted for ethnic minority background, depression in the index pregnancy and self-reported pelvic girdle syndrome. The results for MVPA persisted in the sub-sample of ethnic minority women. 
Limitations: Numbers of cases with PPDS were limited. TheSWAdoes notmeasure water activities. Due to missing data for SWA we used multiple imputations.

Conclusion: Women meeting the physical activity recommendation (>150MVPA min/week) during pregnancy have a lower risk of PPDS compared to women who are not active during pregnancy. 


\section{Physical activity in pregnancy and postpartum depressive symptoms ina multiethnic cohort}

Nilam Shakeel ${ }^{1}$, Kåre Rønn Richardsen ${ }^{2}$, Egil W. Martinsen ${ }^{3}$ Malin Eberhard-Gran 4,5,6 Kari Slinning $^{7}$, Anne Karen Jenum ${ }^{8}$

Introduction:There is strong evidence that postpartum depression is associated with adverse health effects in the mother and infant. Fewstudies haveexplored associations between physical activity in pregnancy and postpartum depression. We aimed to investigate whether physical activity during pregnancy was inversely associated with postpartum depressive symptoms, PPDS in a multiethnic sample.

Method: Population-based, prospective cohort of 643 pregnant women (58 \% ethnic minorities) attending primary antenatal care from early pregnancy to postpartum in Oslo between 2008 and 2010. Data on demographics and health outcomes were collected during standardized interviews. PPDS was defined by a sum score $\geq 10$ from the Edinburgh Postnatal Depression Scale (EPDS), 3 months after birth. Physical activity was recorded with Sense Wear ${ }^{\text {TM }}$ Pro3 Armband (SWA) in gestational week 28 and defined as moderate-tovigorous intensity physical activity (MVPA) accumulated in bouts $\geq 10$ minutes.

Results: Women who accumulated $\geq 150$ MVPA minutes/week had significantly lower risk $(\mathrm{OR}=0.2,95 \% \mathrm{Cl}: 0.06-0.90)$, forPPDScompared to those whodidnotaccumulateany minutes/week of MVPA, adjusted for ethnic minority background, depressive symptoms in indexpregnancy in the indexpregnancy and self-reportedpelvicgirdlesyndrome. The results for MVPA persisted in the sub-sample of ethnic minority women. 
Limitations: Numbers of cases with PPDS were limited. The SWAdoes not measure water activities. Due to missing data for SWA we used multiple imputations.

Conclusion: Women meeting the physical activity recommendation (>150 MVPAmin/week) during pregnancy have a lower risk of PPDS compared to women who are not active during pregnancy.

\section{Keywords}

Postpartum depression; Postpartum depressive symptoms; Physical activity; Ethnic minorities; Objectively recorded moderate to vigorous physical activity;

\section{Abbreviations}

PPD: postpartum depression; PPDS: postpartum depressive symptoms; SEP: socioeconomic position; MVPA: moderate-to-vigorous intensity physical activity; $\mathrm{CHC}$ : child health clinic; EPDS: Edinburgh Postnatal Depression Scale;SWA:SenseWearTM Pro3Armband;DAG: Directed AcyclicGraph;OR:odds ratio; Cl:confidence interval;BDI:BeckDepression inventory; RCT: randomized controlled trial

\section{Introduction}

Depression in the postpartum period has been identified as the most common complication of childbearing years [1], with an estimated prevalence of 10-20\% in Westerncountries [2] [3]. Some women have continuation of depression that presented in pregnancy. For others it 
is of new onset. Women who experience perinatal complications or have a co-existing chronic disease have an even higher risk for postpartum depression [4]

The Diagnostic and Statistical Manual of Mental Disorders, (DSM-5-2013) [5] does not define postpartum depression (PPD) as a specific diagnostic category, but does allow for the addition of a "peripartum onset specifier" if depression starts in pregnancy or within four weeks afterdelivery [2,6]. Manifestation of PPD can be mild to severe, anditis associated with impaired maternal psychological, emotional and cognitive functions [6].

Furthermore, PPD may negatively affect the relationships among family members and the attachment between the baby and the mother, which may have long-term negative influence on the child [7-11]

Immigrantwomen in WesternEuropean countries often face multiplechallenges due to stressors related to migration, integration, poor housing conditions and racism [12-14]. This makes them vulnerable to mental health problems like depression [15]. Ethnic minority women in Western countries have higher prevalence of PPD compared to native Western European[3]. We have earlier reported higher rates of depressive symptoms in pregnancy among ethnic minorities compared to Western Europeans [16]. Risk factors associated with depression in pregnancy in that study included a low level of socioeconomic position (SEP), previous history of depression, recent adverse life events and a low level of integration.

While it is well documented that physical activity outside pregnancy prevents depression $[17,18]$, evidence on preventive effects of physical activity in pregnancy on PPD and postpartum depressive symptoms (PPDS) is inconclusive [19-21]. 
We identified only one RCT study designed to determine the effect of a supervised exercise program implemented in pregnancy; no protective effect against depressive symptoms was found three months after birth [19].

Mixed findings have been reported in observational studies based on self-reported physical activity [20,21]. A lower risk of depression was reported in women who were moderately physically active in the $3^{\text {rd }}$ trimester compared to inactive women [21]. In contrast, in a retrospective study, no significant association was observed between physical activity in pregnancy and EPDS score three months after birth [20]. The use of self-reported physical activity in these two latter studies makes the results prone to measurementerror, and to subsequently biased estimates. In addition, the study samples in above-mentioned studies have limited external validity with respect to multi-ethnic populations.

In addition to established forms of treatment for depressive symptoms in the postpartum period, otherpotentially feasibleand cost-effectivestrategies likephysicalactivity during pregnancy may represent a complementary strategy to attenuate depressive symptoms and reduce reliance on pharmaceutical treatment $[22,23]$. Currently, healthy pregnant and postpartum women are recommended to undertake a minimum of 150 min of moderate-tovigorous intensity physical activity (MVPA) per week for health benefits [24].

Our primary aim was to investigate if higher levels of MVPA in pregnancy were associated with reduced risk of postpartum depressive symptoms, PPDS, overall and in a sub-sample of ethnicminority women, as we hypothesized that MVPAduring pregnancy was inversely associated with risk of PPDS. 


\section{Method}

\section{Design, study population and setting}

This study is part of the prospective STORK Groruddalen Cohort Study of healthy pregnant women, based on datacollected at threepublic Child Health Clinics $(\mathrm{CHC})$ in multi-ethnic city districts in Oslobetween2008and 2011 [25]. Relevant information material suchas invitation leaflets and questionnaires were translated from Norwegian into Arabic, English, Sorani, Somali, Tamil, Turkish, Urduand Vietnamese and quality-checked by bilingual health professionals. Datawere collected at three study visits (at inclusion in mean gestational week 15; in gestational week 28; and 3 months after birth). Demographic and health questionnaire datawere collected during standardizedinterviews. Physical activity was objectively recorded.

Women were eligible for participation if they: 1) lived in one of the study districts; 2) planned to give birth at one of two study hospitals; 3 ) were $<20$ weeks gestation at inclusion; 4) could communicate in either of the nine questionnaire languages; and 5) were able to give informed written consent. Women known to have diabetes and/or other diseases necessitating intensive hospital follow-up during pregnancy were excluded. The inclusion rate was 74\% (range 64-83\% among ethnic groups), and the participating women were found to berepresentative ofpregnantwomeninthelargestethnicgroups attending the Child Health Clinics [25, 26]. Informed consent was obtained from all individual participants included in the study.

\section{Primary outcome variable - postpartum depressive symptoms}

We measured postpartum depressive symptoms approximately three months after birth using the validated screening instrument The Edinburgh Postnatal Depression Scale (EPDS) 
$[27,28]$. EPDS contains ten items scored on a four-point ordinal scale, and ityields a sum score between 0 and 30 . A high score indicates depression, but in clinical practice, we need a diagnosticinterviewto set the diagnosis depression. AnEPDS score $\geq 10$ was used as indicative of an increased depression risk herby called PPDS, as this cut-off point has been used previously in several epidemiological studies [16, 29-31].

We used eight official translations of the EPDS (Norwegian, Arabic, English, Somali, Tamil, Turkish, Urdu and Vietnamese). In addition, we used a version in Sorani, translated by the City Services Department's Interpreting Service in Oslo, for this study. All exceptthe Somalian and Sorani versions have been validated [32].

\section{Main exposure - objectively measured physical activity}

We collected physical activity data objectively with the activity monitor Sense Wear ${ }^{\mathrm{TM}}$ Pro3 Armband (SWA) (Body Media Inc., Pittsburgh, Pennsylvania, USA) [33] in gestational week 28.-The SWA is a multi-phasic monitor which incorporates information from accelerometerswith data from sensors for heat flux, skin temperature and galvanic skin response [34]. Women were asked to wear the SWA across the right triceps brachia over 4-7 days following the study visit, and remove it only if performing water activities. We downloaded raw physical activity dataintegrated into60-secondepochs with the manufacturer's software (Sense Wear ${ }^{\text {TM }}$ Professional Research Software Version 6.1, Body Media Inc.). A validSWA day was defined as $\geq 19.2 \mathrm{~h}$ of SWA wear time, and physical activity data from a single participant was deemed eligible if $\geq 2$ valid SWA days were recorded [35].

SWA provides valid measures of energy expenditure during free-living activities during pregnancy [33]. 
MVPA was restricted to bouts $\geq 10$ minutes at intensities $\geq 3$ metabolic equivalents (METs) (1 $\left.\mathrm{MET}=3.5 \mathrm{O}^{2} \mathrm{~kg}^{-1} \cdot \mathrm{min}^{-1}\right) \cdot \mathrm{MVPA}$ in bouts were extracted with SQL Server Management Studio (Microsoft $\AA$ ) andSQLServerExpressversion 11.0.50580 (Microsoft $®$ ). MVPAwas treatedas an ordinal variable with four levels: 1) 0 minutes per week, 2) 1-74 minutes per week, 3) 75-149 minutes/week, and 4) $\geq 150$ minutes/week. These categories were selected to ensure a certain number of cases in each activity group. We defined the most active group as women who accumulated $\geq 150$ minutes to ensure that one group represented women who met the recommended level of MVPA.

\section{Other potentially confounding variables}

We defined ethnicity by the participant's country of birth, or her mother's country of birth if her mother was born outside Europe or North America. In analyses, ethnicity was treated as abinary variable (ethnic minority women andWestern-European women) [16]. Maternal age at inclusion was treated as a continuous variable.

Data on socioeconomic position (SEP) collected at inclusion were expressed as a component score (Crohnbach's $\alpha>0.7$ ) extracted using principal components analysis [36], reflecting educational level, occupational class, employmentstatus, renting tenure androoms per person in the household. Higher SEP scores reflected a higher socio-economic position. The SEP scores were normally distributed and treated as a continuous measureand binary variable.

Data on adverse life events were collected at visit 1 and 2 , referring to the preceding six months at each visit. Items reflected seven events (serious illness of self; serious illness in close family; death in close family; divorce/separation; unemployment; major concern for children; other major adverse event) with the response options being "yes" and "no". In 
analyses, thetotal number of events from visit 1 and 2 wastreated as an ordinal variable (no events; one event; two or more events).

Depressive symptoms inindex pregnancy was assessed using the EPDS tool atvisit2 (gestational week 28) and defined as EPDS score $\geq 10$.

Information on history of depression was obtained postpartumusing Kendler`s lifetime major depression scale, which assesses lifetime history of major depression based on the DSM-IV criteria. This five-itemscalecoverssadness, appetitechanges, lack of energy, selfblame and concentration problems. The response categories were "yes" and "no". Prior depression was defined as having had at least two simultaneously concurrent symptoms with duration of at least two weeks in addition to a sad mood [37, 38].

Self-reported pelvic girdle syndrome (PGS), was identified as a possible confounder [39] and defined as having pain from both iliac-sacral joints and the pubic symphysis [40].

Parity, age at inclusion, marital status and educational level were used for descriptive purposes. Parity was categorized as nulliparous or multiparous, marital status was categorized as living with a partner or living without a partner and education was categorized into four categories: $<10$ years schooling, secondary level 10-12 years, up to 4 years of further education and University/College education.

\section{Statistical analysis}

Descriptivestatistics are provided as mean (SD) and frequency (\%) as appropriate. To compare groups, chi-square tests were used for categorical data and T-tests for continuous variables. 
We used a Directed Acyclic Graph (DAG) (figure 1) in the model building process as a flowchart to visualize the causal network, linking MVPA and PPDS, and identify confounders [41]-that should be adjusted for to attain unbiased and more precise estimates [42]. Thereafter, we performed multiple logistic regression analyses to explore the association between MVPA and PPDS. Due to the rule of degrees of freedom, the number of confounders we could adjust forwas limited as the sample contains only 60 cases with PPDS. A reduction of confounders was performed as we fitted the model using the "purposeful selection" approach [43] in which non-significant cofactors were removed by backward selection ifthey did not change the value of the mainestimate. In model 1, we adjusted for ethnicity, age, SEP, adverse life events, depressive symptoms in index pregnancy, self-reported pelvicgirdle syndromeand sedentary time. The final model contained MVPA, ethnicity, depressive symptoms in index pregnancy and self-reported pelvic girdle syndrome.

Missing data on exposure or covariates were identified in data on MVPA, sedentary time, adverse lifeevents, depressivesymptoms in indexpregnancy, self-reported pelvic girdle syndrome and history of depression (Table 1). Missing MVPA values were significantly predicted by non-Western ethnicity. Thus, the inclusion of ethnicity among the variables, the imputation model supported the plausibility of the missing at randomassumption [44]. We included ethnicity, EPDS at visit3 (outcome in analytic model), age, BMl in the imputation model and imputed the following variables: MVPA/sedentary time, SEP, adverse life events, depressive symptoms in index pregnancy, and pelvic girdle pain. We generated 25 imputed datasets to obtain pooled estimates of the overall measures of association [44] using the automatic mode in SPSS. The imputed values were used in the primary multiple logistic regression analyses. 
The analyses were repeated in a sub-sample of ethnic minority women in accordance with ouraim. Wealsoperformed asensitivity analysisonthe total samplebasedoncomplete cases.

The statistical significance level was set to $p<0.05$. SPSS version25 (IBM SPSS statistics, NY, USA) was used for all analyses.

\section{Study sample}

Our sample consisted of 643 women with a valid EPDS score from postpartum, $78 \%$ of the total sample of 823 included in the STORK Groruddalen study [16].

\section{Results}

\section{Sample characteristics}

In our sample, we had 60 cases (9.3\%) with PPDS and $42 \%$ of the women were ethnic minorities. The mean age in the whole sample was 30 years, $54 \%$ were nulliparous, $37 \%$ had Iow SEP and $15 \%$ had less than 10 years education.

Among women who recorded no MVPA minutes/week in bouts $>10$ minutes, a larger proportion were multiparous, had ethnic minority background, lower socioeconomic position (SEP) and fewer had higher education at university and college level, compared to those with $\geq 150$ MVPAminutes/week (Table1). Morewomen in the group withnoMVPA minutes/week had depressive symptoms in index pregnancy and PPDS. The average age and number of adverse life events, however, were similar for both groups.

\section{$\underline{\text { Association between physical activity and postpartum depression }}$}


In bivariate logistic regression analysis with imputed values, PPDS were associated with inactivity, measured as no MVPA minutes/week, ethnic minority background, adverse life events, low socioeconomic position, history of depression and depression in index pregnancy, but not with pelvic girdle pain (Table 2).

Further, we observed that women who met the physical activity recommendation $(\geq 150$ MVPA min/week) had a significantly lower risk of PPDS (OR $=0.2 ; 95 \%$ Confidence interval (CI): 0.06-0.63), (Table 3: unadjusted values). After adjustment for ethnicity, depressive symptoms in index pregnancy and self-reported pelvic girdle syndrome, the association remained significant $(\mathrm{OR}=0.2 ; 95 \% \mathrm{Cl}: 0.06-0.90)$ (Table 3). Analyses based on complete cases yielded similar results (added as appendix, table4).

We then repeated the analyses in the subgroup of ethnic minority women only. Women who met the physical activity recommendation had a significantly lower risk of PPDS (OR: 0.2 see appendix, table 5). Again, similar results were found in the analysis for complete cases (added as appendix, table 6).

\section{Discussion}

To the best of our knowledge, this is the only study exploring the association between objectively recorded physical activity in pregnancy and PPDSin a multiethnic cohort. We observed that women who performed $\geq 150$ minutes per week of moderate to vigorous physical activity in bouts $\geq 10$ min had lower risk of PPDS, compared to those who did not accumulate any physical activity of at least moderate intensity. This finding was replicated in a sub-sample of ethnic minority women. 
Very few studies have explored the relationship between physical activity in pregnancy and symptoms of depression after birth like us, and findings are contradictory [19-21]. Only one smallstudy with high-income Caucasianwomenfound an inverseassociationbetween physical activity and PPDS symptoms [45].

Incontrast to ourstudy, one longitudinal prospective cohortstudy by Demisse etal. did not find any significant association between MVPA in pregnancy and PPDS in the full sample nor in analyses stratified by ethnicity (white, black and others) [46]. But this study used selfreported measures forphysical activity which may have led to recalland social desirability biases. Further, the sample was self-selected and therefore not representative for the source population.

A review by Teychenne [47] including cross sectional studies, randomized controlled trials (RCTs) and longitudinal cohort studies, reported that out of the ten high quality observational studies exploring the effect on PPDS, only four examined physical activity in pregnancy, all byself-reports. Timing of physical activity, measures and findings were inconsistent. This study concluded that physical activity in pregnancy is importantfor reducing the risk of postnatal depression.

Recently, two systematic reviews and meta-analyses addressing exercise both in pregnancy and postpartum and their association with both PPDS and postpartum depression respectively have been published [1,48]. Poyatos-Leon and colleagues [1] included 12RCTs, showing that physical activity was associated with a lower incidence of depressive symptoms postpartum. However, in only two of these studies the exercise program started in pregnancy. For the rest of the studies, the physical activity programs for the intervention group were conducted only during the postpartum period. Generally, the quality of most of the studies included in this meta-analysis was low, with high risk of bias. 
Pritchett, Daley and Jolly (2017) [48] found 13RCTs, and concluded that exercisemight reduce depressivesymptoms. However, all of these studiestargeted womenfirst in the postpartum period with interventions to promote physical activity. Six studies assessed the general postpartum population regardless of whether they had scored compatible with depression or not, while the other studies included postpartum women with high symptom scores on depression defined by different assessment tools (EPDS, diagnostic interviews using ICD-10/DSM-4 criteria and Beck depression inventory (BDI). A direct comparison with other studies is difficult as there is a dearth in the literature regarding studies exploring the same research question as ours.

However, we consider the dearth of studies of protective effects of physical activity during pregnancy makes our findings highly relevant.

Furthermore, only few of the studies carried out in Western Europe reported if ethnic minority women were included, but most included only women who spoke the majority language. None of the studies included translators, which indicate that those with insufficient hostlanguage skills and those who are newly arrived to the hostcountry most probably were excluded. Hence, the results are not generalizable to less integrated ethnic minority women. In contrast, in our study $58 \%$ of the Stork-Groruddalen Cohort was ethnic minority women, some of them had recently arrived in Norway and had limited command of the Norwegian language. Thus, the current cohort is more representative of current multi-ethnicpopulations in many Europeancountries. Anovel and importantfinding is the relative risk reduction associated with achieving $\geq 150 \mathrm{MVPA}$ min/week in the sub-sample of ethnic minority women.

We do not know how the benefits of exercise are mediated. Generally, the effects of exercise on depression are widely known but poorly understood [49] and both psychological 
and neurobiological mechanisms are suggested. Relapse is common in depressive disorders, and continuous physical activity maybe useful in the efforts to preventfuture episodes. In general, a positive effect of exercise on depression may be long lasting

\section{Strengths and limitations}

Our study has several strengths. This is a population-based prospective cohort study with a high attendancerate and arepresentative multi-ethnicsample. The prospectivedesign reduced the likelihood of recall bias. We have succeeded in including illiterate and recently immigrated women by adapting the study methods to the needs of these women [25]. We used EPDS, which is a validated screening tool, with good agreement with the DSMIV criteriafor major depression (100\% sensitivity and $87 \%$ specificity) when using EPDSscores $\geq 10$ as the cut-off [29,31]. Furthermore, we used objectively recorded physical activity, which provides more valid estimates of habitual physical activity than self-report methods. The analytic models included potential confounders identified by a DAG, reducing the risk that the association between MVPA and PPDS is spurious.

Nevertheless, our study has some limitations. First, the cross-cultural validity of the EPDS is a concern. The expression of depression may differ according to cultural context; however, the EPDS has good psychometric properties when tested in different cultures [32, 50-53]. Differentcut-offvalueshave been suggested whenEPDS is translated and validatedinto other languages [32], and it is recommended that health care professionals review the summary of the research to best match the characteristics of their population. As we wanted to compare thesymptom load in differentethnic groupsinEurope living in the same residential area, we used the same cut-off level for all women. 
Second, the physical activity monitor does not measure water activities. While the SWA provides valid measures of energy expenditure during pregnancy for a range of physical activity types, the validity during bicycling during pregnancy is unclear [33]. Unpublished datafromourstudy has shown that very fewwomen were bicycling or participating in water activities during pregnancy. Third, participants' physical activity level may have increased because of wearing the armband; hence, the physical activity estimates may not reflect the true habitual level of physical activity during pregnancy.

Forth, a slight selection bias cannot be ruled out as women with higher SEP, more education and more time spent living with their partner participated compared to those who did not re-attend after their first or second visit. This is often the case in cohort studies with longer follow-up-times. Fifth, although power is limited and we primarily report results based on multiple imputations due to missing, complete cases gave similar results. Lastly, we cannot rule out unmeasured or residual confounding, and cultural factors and traditions may play a role.

\section{Implications for practice and research}

More awareness is needed among health personnel in antenatal and postnatal care about womenat risk forPPDS, and more knowledge aboutthe usefulness of physical activity to reduce the risk of PPDS is warranted among clinicians. The beliefs about physical activity and health in differentculturesvary [32], and in somegroups, rest after birth is considered to be beneficial to health [54]. Hence, culture sensitive preventive measures should contain information on the potential of regularphysicalactivity to reducePPDS, and strategiesfor facilitation of more physical activity during pregnancy in vulnerable groups. Nevertheless, 
there is still a lack of high quality RCT studies using objectively recorded physical activity in pregnancy to determine the effect on PPDS.

\section{Conclusion}

The study suggests that physical activity in late pregnancy may reduce the risk of PPDS. Importantly, the risk reduction was also seen in ethnic minority women.

\section{Acknowledgements}

First, the authors thank all the study participants. We also thank study staff at the CHCs in Stovner, Grorud and Bjerke districts in Oslo for collecting the data. Translation of information material and questionnaires was performed by the City Services Department, The Interpreting and Translating Service in Oslo. A special thank to statistician Ragnhild Sørum Falck who helped/assisted us with statistical analysis.

\section{Authors' contributions}

NS and KRR performed the statistical analysis (except the PCA analyses). NS wrote the first draft-KRR made the figure and did the multiple imputation analysis. KRR also helped revising the first draft of the paper. LScontributed with the data acquisition and performed the PCA analyses on socio-economic and integration variables, ME-G, KS, and EWM contributed with expert knowledge about the EPDS and other instruments to capture depression. AKJ initiated and was the project leader of the STORK Groruddalen study. All authors contributed to the interpretation of data, revised the manuscript critically, checked for clarity and content, and approved the final version. 


\section{Funding}

The STORK Groruddalen research program was primarily funded by the Norwegian Research

Council, with additional funding from The South-Eastern Norway Regional Health Authority, Norwegian Directorate of Health and collaborative partners in The City of Oslo, Stovner, Grorud and Bjerke administrative districts. Nilam Shakeel received funding for a PhD grant through the Norwegian Research Fund for General Practice. The funder had no involvement in any aspects of the study.

\section{Ethics approval and consent to participate}

The study was approved by The Regional Committee for Medical and Health Research Ethics for South Eastern Norway (reference number: 2007.894) and The Norwegian Data Inspectorate. All participants gave their written informed consent.

\section{References}

1. Poyatos-Leon, R., et al., Effects of exercise-based interventions on postpartum depression: $A$ meta-analysis of randomized controlled trials. Birth, 2017. 44(3): p. 200-208.

2. Dennis, C.L. andE. Hodnett, Psychosocial and psychological interventionsfortreating postpartum depression. Cochrane Database Syst Rev, 2007(4):p. Cd006116.

3. Falah-Hassani, K., etal., Prevalence of postpartum depression among immigrant women: A systematic review and meta-analysis. J Psychiatr Res, 2015. 70: p. 67-82.

4. Turner, K., et al., Postpartum depression in women with epilepsy versus women without epilepsy. Epilepsy Behav, 2006. 9(2): p. 293-7.

5. Association., A.P., American Psychiatric Association. Diagnostic and Statistical Manual of Mental Disorders. 5th ed. Washington, DC: American Psychiatric Publishing 2013. 2013.

6. O'Hara, M.W. and J.E. McCabe, Postpartum depression: current status and future directions. Annu Rev Clin Psychol, 2013. 9: p. 379-407. 
7. Stein, A., et al., Effects of perinatal mental disorders on the fetus and child. Lancet, 2014. 384(9956): p. 1800-19.

8. Grace, S.L., A. Evindar, and D.E. Stewart, The effect of postpartum depression on child cognitive development and behavior: A review and critical analysis of the literature. Arch Womens Ment Health, 2003. 6.

9. Hay, D.F., et al., Pathways to violence in the children of mothers who were depressed postpartum. Dev Psychol, 2003. 39(6): p. 1083-94.

10. Hay, D.F., et al., Intellectual problems shown by 11-year-old children whose mothers had postnatal depression. J Child Psychol Psychiatry, 2001. 42(7): p. 871-89.

11. Murray, L., et al., Maternal postnatal depression and the development of depression in offspring up to 16 years of age. J Am Acad Child Adolesc Psychiatry, 2011. 50(5): p. 460-70.

12. Karlsen, S. and J.Y.Nazroo, Relation between racial discrimination, social class, and health among ethnic minority groups. Am J Public Health, 2002. 92(4): p. 624-31.

13. Wittkowski, A., S. Patel, and J.R. Fox, The Experience of PostnatalDepression in Immigrant Mothers Living in Western Countries: A Meta-Synthesis. Clin Psychol Psychother, 2016.

14. Fung, K. and C.L. Dennis, Postpartum depression among immigrant women. Curr Opin Psychiatry, 2010. 23(4): p. 342-8.

15. Williams, D.R., Race, socioeconomic status, and health. The added effects of racism and discrimination. Ann N Y Acad Sci, 1999. 896: p. 173-88.

16. Shakeel, N., et al., A prospective cohort study of depression in pregnancy, prevalence and risk factors in a multi-ethnic population. BMC Pregnancy Childbirth, 2015. 15: p. 5.

17. Mammen, G. and G. Faulkner, Physical activity and the prevention of depression: a systematic review of prospective studies. Am J Prev Med, 2013. 45(5): p. 649-57.

18. FYSS, Yrkesføreningar førfysisk aktivitet, fysisk aktivitet i sjukdomsprevention och sjukdomsbehandling, FYSS

http://www.fyss.se/in-english/chapters-in-fyss/. 2015. 2017.

19. Songoygard, K.M., et al., Does exercise during pregnancy prevent postnatal depression? A randomized controlled trial. Acta Obstet Gynecol Scand, 2012. 91(1): p. 62-7.

20. Ersek, J.L. and L.R.BrunnerHuber, Physical activity priorto and during pregnancy and risk of postpartum depressive symptoms. J Obstet Gynecol Neonatal Nurs, 2009. 38(5): p. 556-66.

21. Nordhagen, I.H. and J.Sundgot-Borgen, [Physical activity among pregnant women in relation to pregnancy-related complaints and symptoms of depression]. Tidsskr Nor Laegeforen, 2002. 122(5): p. 470-4.

22. Saligheh, M., et al., Can exercise or physical activity help improve postnatal depression and weight loss? A systematic review. Arch Womens Ment Health, 2017.

23. Brandlistuen, R.E., et al., Association of prenatal exposure to benzodiazepines and child internalizing problems: A sibling-controlled cohort study. PLoS One, 2017. 12(7): p.e0181042.

24. Norweigan directorate of halth, h.N., Anbefalinger fysisk aktivitet, gravide og etter fødsel. www.helsedirektoratet.no, 2016.

25. Jenum, A.K., et al., The STORK Groruddalen research programme: A population-based cohort study of gestational diabetes, physical activity, and obesity in pregnancy in a multiethnic population. Rationale, methods, study population, and participation rates. Scand J Public Health, 2010. 38(5 Suppl): p. 60-70.

26. Jenum, A.K., et al., Impact of ethnicity on gestational diabetes identified with the WHO and the modified International Association of Diabetes and Pregnancy Study Groups criteria: a population-based cohort study. Eur J Endocrinol, 2012. 166(2): p. 317-24.

27. Cox, J.L., J.M. Holden, and R. Sagovsky, Detection of postnatal depression. Development of the 10-item Edinburgh Postnatal Depression Scale. Br J Psychiatry, 1987. 150: p. 782-6.

28. Murray, L. and A.D. Carothers, The validation of the Edinburgh Post-natal Depression Scale on a community sample. Br J Psychiatry, 1990. 157: p. 288-90. 
29. Harris, B., et al., The use of rating scales to identify post-natal depression. Br J Psychiatry, 1989. 154: p. 813-7.

30. Hewitt, C., et al., Methods to identify postnatal depression in primary care: an integrated evidence synthesis and value of informationanalysis. Health TechnolAssess, 2009.13(36):p. 1-145, 147-230.

31. Eberhard-Gran, M., etal., The Edinburgh PostnatalDepression Scale: validation in a Norwegian community sample. Nord J Psychiatry, 2001. 55(2): p. 113-7.

32. Dr Jann Marshall, Edinburgh Poastnatal depression scale (EPDS) Translated Versions

https://www.mcpapformoms.org/Docs/Edinburgh\%20Depression\%20Scale\%20Translated\%20Govern ment\%20of\%20Western\%20Australia\%20Department\%20of\%20Health.pdf. 2006.

33. Berntsen, S., S.N. Stafne, and S. Morkved, Physical activity monitor for recording energy expenditure in pregnancy. Acta Obstet Gynecol Scand, 2011. 90(8): p. 903-7.

34. Shephard, R.J., \& Tudor-Locke, C. , The Objective Monitoring of Physical Activity: Contributions of Accelerometry to Epidemiology, . Exercise Science and Rehabilitation: Springer., 2016.

35. Herrmann, S.D., et al., Impact of accelerometer wear time on physical activity data: a NHANES semisimulation data approach. Br J Sports Med, 2014. 48(3): p. 278-82.

36. Sommer, C., et al., Ethnic differences in maternal dietary patterns are largely explained by socio-economic score and integration score: a population-based study. Food Nutr Res, 2013. 57.

37. Kendler, K.S., et al., The lifetime history of major depression in women. Reliability of diagnosis and heritability. Arch Gen Psychiatry, 1993. 50(11): p. 863-70.

38. Kjeldgaard, H.K., et al., History of depression and risk of hyperemesis gravidarum: a population-based cohortstudy. Archives of Women's Mental Health,2017:p. 1-8.

39. Bjelland, E.K., et al., The effect of emotional distress on persistent pelvic girdle pain after delivery: a longitudinal population study. Bjog, 2013. 120(1): p. 32-40.

40. Albert, H., M. Godskesen, andJ.Westergaard, Prognosisinfoursyndromes of pregnancyrelated pelvic pain. Acta Obstet Gynecol Scand, 2001. 80(6): p. 505-10.

41. Hernan, M.A., et al., Causal knowledge as a prerequisite for confounding evaluation: an application to birth defects epidemiology. Am J Epidemiol, 2002. 155(2): p. 176-84.

42. Foraita R, S.J., \& Zeeb, H., Directed Acyclic Graphs. In W. Ahrens \& I. Pigeot (Eds.), Handbook of Epidemiology (pp. 1481-1519). New York, NY: Springer New York. 2014.

43. Marit B., S.L., Petter L. , Medical statistics in clinical and epidemiological research, book. 2012.

44. Sterne, J.A.C., etal., Multiple imputation for missing data in epidemiological and clinical research: potential and pitfalls. BMJ, 2009.338.

45. Sexton, M.B., et al., Predictors of recovery from prenatal depressive symptoms from pregnancythrough postpartum. JWomens Health (Larchmt), 2012.21(1):p. 43-9.

46. Demissie, Z., et al., Physical activity during pregnancy and postpartum depressive symptoms. Midwifery, 2013. 29(2): p. 139-147.

47. Teychenne, M. and R. York, Physicalactivity, sedentary behavior, and postnatal depressive symptoms: a review. Am J Prev Med, 2013. 45(2): p. 217-27.

48. Pritchett, R.V., A.J.Daley, andK. Jolly, Does aerobic exercise reduce postpartum depressive symptoms? a systematic review and meta-analysis. BrJGenPract,2017.67(663):p.e684e691.

49. Daley, A.J., C. Macarthur, and H. Winter, The role of exercise in treating postpartum depression: a review of the literature. JMidwifery Womens Health, 2007.52(1):p.56-62.

50. Husain, N., et al., Prevalence and psychosocial correlates of perinatal depression: a cohort study from urban Pakistan. Arch Womens Ment Health, 2011. 14(5): p. 395-403. 
51. Benjamin D, C.A., Annie IK, Prasad J, Jacob KS, Validation of the tamil version of Edinburgh post-partum depression scale. the journal of obstetrics and gynecology of India, 2005. 55(may/june 2005): p. 242-243.

52. Aydin, N., et al., Validation of the Turkish version of the Edinburgh Postnatal Depression Scale among women within theirfirst postpartum year. Soc Psychiatry Psychiatr Epidemiol, 2004. 39(6): p. 483-6.

53. Berle, J.O., et al., Screening for postnatal depression. Validation of the Norwegian version of the Edinburgh Postnatal Depression Scale, and assessment of risk factors for postnatal depression. J Affect Disord, 2003. 76(1-3): p. 151-6.

54. Eberhard-Gran, M., et al., Postnatal care: a cross-cultural and historical perspective. Arch Womens Ment Health, 2010. 13(6): p. 459-66. 


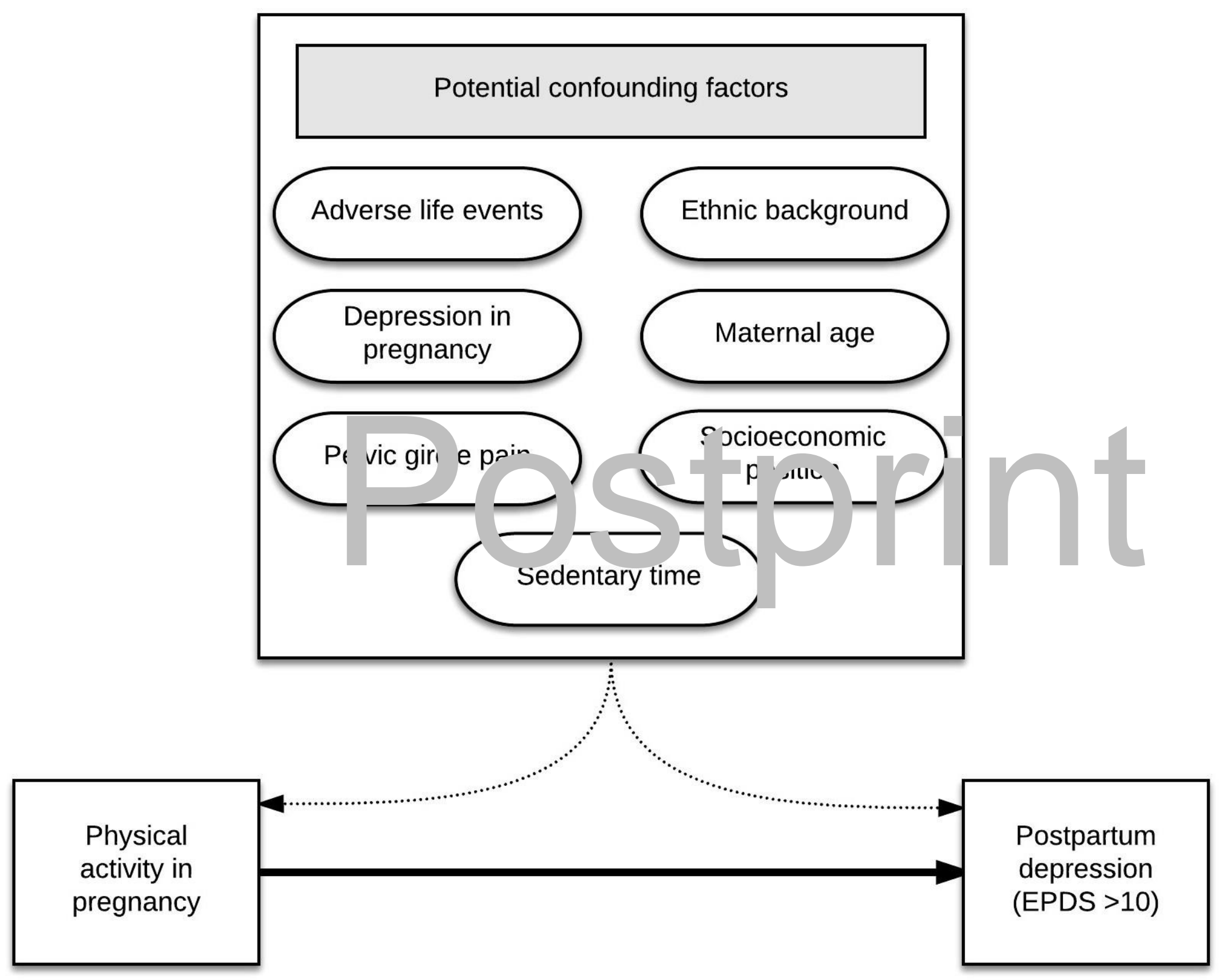


Table 1, Sample Characteristics

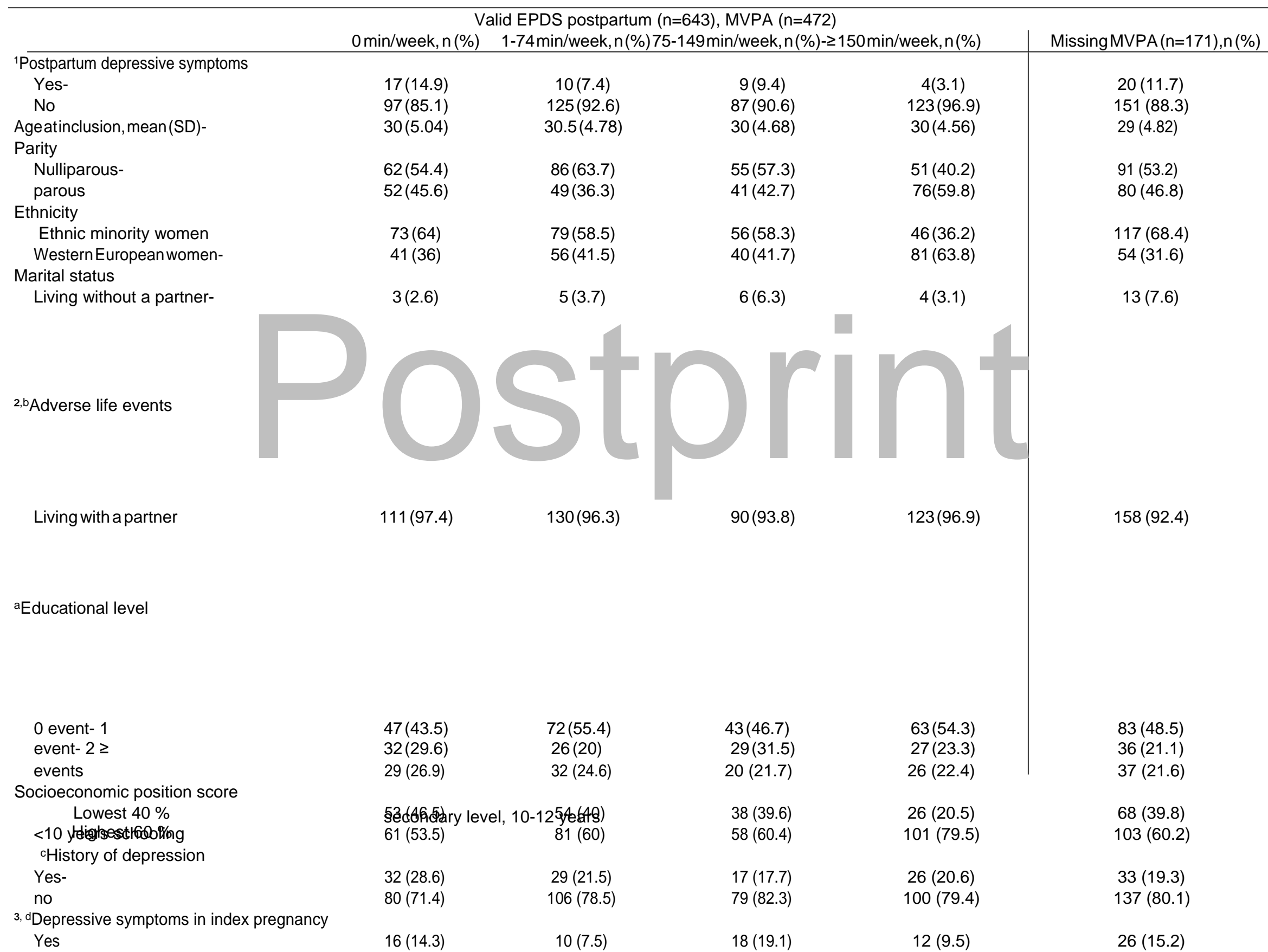


up to 4 years of further education-

$19(16.7)$

$20(14.9)$

4(14.6)

$12(9.4)$

31 (18.1)

$53(46.5)$

$61(45.5)$

$35(36.5)$

$38(29.9)$

$33(28.9)$
$30(31.3)$

$51(40.2)$
45 (26.3) 

${ }^{1}$ EPDS $\geq 14$ weeks postpartum ${ }^{2}$ From inclusion to gestational week $28{ }^{3}$ EPDS $\geq 10$ in gestational week $28{ }^{4}$ pain both ilio-sacral joints and the pubic symphysis- 
aMissing $n=1$, ${ }^{b}$ Missing $n=26$, ${ }^{c}$ Missing $n=3$, ${ }^{d}$ Missing $n=7$, eMissing $n=7$ 
Table 2

Characteristic of women with and without postpartum depression, Values are $\mathrm{n}(\%)$ if not stated otherwise

\begin{tabular}{|c|c|c|c|}
\hline & $\begin{array}{c}\text { Depression, } \\
\mathbf{N}=60 \\
\mathrm{n}(\%)\end{array}$ & $\begin{array}{c}\text { No depression, } \\
\mathbf{N}=583 \\
\mathrm{n}(\%)\end{array}$ & P-value \\
\hline \multicolumn{4}{|l|}{${ }^{1}$ PA during pregnancy } \\
\hline 0 min a week & $33(56.9)$ & $163(31.8)$ & 0.001 \\
\hline 1-74 min a week & $10(17.3)$ & $128(25)$ & \\
\hline 75-149 min a week & $10(17.3))$ & $91(17.8)$ & \\
\hline$>150$ min aweek & $5(8.6)$ & $130(25.4)$ & \\
\hline Ethnic minority women & 47 (78.3) & $324(55.6)$ & $<0.01$ \\
\hline Western European women & $13(21.6)$ & 259 (44.4) & \\
\hline Age at inclusion, mean (SD) & $28.8(4.47)$ & $30.0(4.81)$ & 0.07 \\
\hline \multicolumn{4}{|l|}{ Parity } \\
\hline Nulliparous & $27(45)$ & $271(46.5)$ & 0.83 \\
\hline parous & $33(55)$ & $312(53.5)$ & \\
\hline \multicolumn{4}{|l|}{${ }^{2}$ Marital status } \\
\hline Living without a partner & $4(6.8)$ & $30(5.2)$ & 0.61 \\
\hline Living with a partner & $55(93.2)$ & 549 (94.8) & \\
\hline \multicolumn{4}{|l|}{ Educational level } \\
\hline$<10$ years schooling & $12(20)$ & $84(14.5)$ & 0.44 \\
\hline secondary level, $10-12$ years & $26(43.3)$ & $223(38.5)$ & \\
\hline up to 4 years of further education & $15(25)$ & $181(31.3)$ & \\
\hline university/college & & & \\
\hline \multicolumn{4}{|l|}{${ }^{3}$ Adverse life events } \\
\hline 0 events & $19(33.9)$ & $283(53)$ & $<0.001$ \\
\hline 1 event & $11(19.7)$ & $136(25.5)$ & \\
\hline $2 \geq$ events & $26(46.4)$ & 115 (21.5) & \\
\hline \multicolumn{3}{|l|}{ Socioeconomic position score } & $<0.001$ \\
\hline Lowest $40 \%$ & $35(58.3)$ & 204 (35) & \\
\hline Highest $60 \%$ & $25(41.7)$ & $379(65)$ & \\
\hline \multicolumn{3}{|l|}{${ }^{4}$ History of depression } & $<0.001$ \\
\hline Yes & $29(49.2)$ & $108(18.6)$ & \\
\hline no & $30(50.8)$ & $472(81.4)$ & \\
\hline \multicolumn{4}{|l|}{$\begin{array}{l}{ }^{5} \text { Depressive symptoms in index } \\
\text { pregnancy }\end{array}$} \\
\hline Yes & $23(41.1)$ & $59(10.4)$ & $<0.001$ \\
\hline No & $33(58.9)$ & 509 (89.6) & \\
\hline \multicolumn{4}{|l|}{$\begin{array}{l}\text { 'Self-reported pelvic girdle } \\
\text { syndrome }\end{array}$} \\
\hline Yes & $16(27.6)$ & $116(20.5)$ & 0,208 \\
\hline No & $42(72.4)$ & $450(79.5)$ & \\
\hline
\end{tabular}

'Physical activity in gestational week28, ${ }^{2}$ Exact fisher's test used, ${ }^{3}$ From inclusion to gestational week $28,{ }^{4}$ Data collected postpartum, ${ }^{5}$ EPDS $\geq 10$ ingestational week $28,{ }^{6}$ pain both ilio-sacral joints and the pubic symphysis 
Table 3,

Odds Ratios (OR) for postpartum depressive symptoms in unadjusted and adjusted multiple regression analysis.

\section{Unadjusted values}

OR $\quad 95 \% \mathrm{Cl}$

1MVPA late pregnancy, 0 min a week=ref.

$$
\text { 1-74 } \min
$$

$75-149$ min

$\geq 150 \mathrm{~min}$

\subsection{3-1.01}

$0.24-1.18$

$0.06-0.63$

\section{Adjusted values ${ }^{2}$}

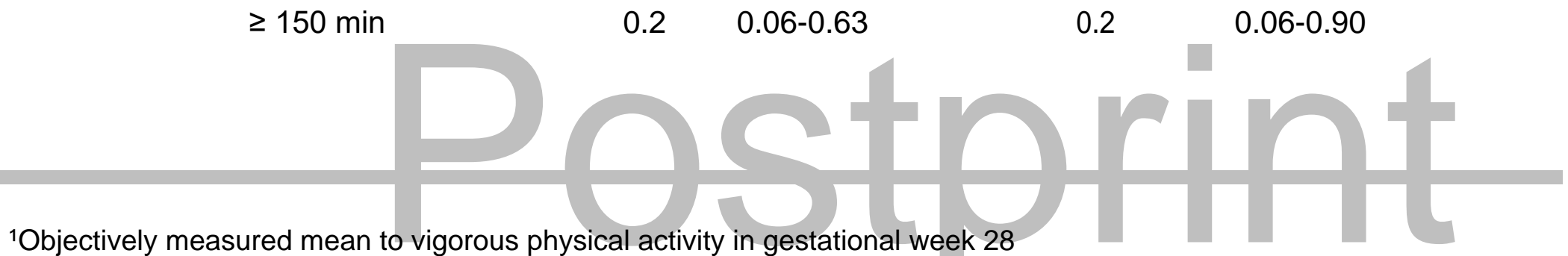

1 Objectively measured mean to vigorous physical activity in gestational week 28

${ }^{2}$ Adjusted for ethnicity, depressivesymptoms EPDS $\geq 10$ ingestational week28andself-reported pelvic girdle syndrome in pregnancy week28 
Appendix, Table 4

Odds Ratios (OR) for postpartum depressive symptoms in unadjusted and adjusted multiple regression analysis.

Complete cases

\begin{tabular}{ccccc}
\hline & \multicolumn{2}{c}{ Unadjusted values } & \multicolumn{2}{c}{ Adjusted values $^{2}$} \\
& OR & $95 \% \mathrm{Cl}$ & $\mathrm{OR}$ & $95 \% \mathrm{Cl}$ \\
\hline${ }^{1}$ MVPA late pregnancy, 0 min a week=ref. & & & & \\
$1-74 \mathrm{~min}$ & 0.5 & $0.20-1.04$ & 0.6 & $0.26-1.56$ \\
$75-149 \mathrm{~min}$ & 0.6 & $0.25-1.49$ & 0.6 & $0.21-1.42$ \\
$\geq 150 \mathrm{~min}$ & 0.2 & $0.06-0.57$ & 0.3 & $0.08-0.88$ \\
& & &
\end{tabular}

${ }^{1}$ Objectively measured mean to vigorous physical activity in gestational week 28

${ }^{2}$ Adjusted for ethnicity, depressivesymptoms EPDS $\geq 10$ ingestational week28andself-reported pelvic girdle syndrome in pregnancy week28 
Ethnic minorities only

Imputed values with postpartum depressive symptoms, PPDS 3 months after birth,

PPDS defined asEPDS $\geq 10$

Unadjusted and adjusted values

\begin{tabular}{cccccccc}
\hline & \multicolumn{3}{c}{ Unadjusted values } & & 1Adjusted values \\
& & & & & \\
& OR & $95 \% \mathrm{Cl}$ & P-value & OR & $95 \% \mathrm{Cl}$ & P-value \\
\hline MVPA late pregnancy, 0 min week=ref & & & & & & \\
$1-74$ min & 0.4 & $0.20-0.94$ & 0.034 & 0.5 & $0.22-1.17$ & 0.106 \\
$75-149$ min & 0.6 & $0.25-1.32$ & 0.187 & 0.5 & $0.18-1.13$ & 0.089 \\
$\geq 150$ min & 0.2 & $0.06-0.55$ & 0.002 & 0.2 & $0.06-0.59$ & 0.004 \\
& & & & & & \\
\hline
\end{tabular}

' Adjusted for depressive symptoms in index pregnancy and self-reprted pelvic girdle syndrom, PGS 
Table 6

Ethnic minorities only

Complete case analysis with postpartum depressive symptoms, PPDS 3 months after birth,

PPDS defined asEPDS $\geq 10$

Unadjusted and adjusted values

Unadjusted values

${ }^{1}$ Adjusted values

\begin{tabular}{ccccccc} 
& $\mathrm{OR}$ & $95 \% \mathrm{Cl}$ & $\mathrm{P}$-value & $\mathrm{OR}$ & $95 \% \mathrm{Cl}$ & $\mathrm{P}$-value \\
\hline MVPA late pregnancy, 0 min a week=ref & & & & & & \\
1 1-74 $\min$ & 0.4 & $0.15-0.98$ & 0.046 & 0.5 & $0.18-1.35$ & 0.167 \\
$75-149 \mathrm{~min}$ & 0.6 & $0.21-1.47$ & 0.233 & 0.5 & $0.19-1.50$ & 0.229 \\
$\geq 150 \mathrm{~min}$ & 0.09 & $0.01-0.68$ & 0.020 & 0.1 & $0.01-0.82$ & 0.031
\end{tabular}

'Adjusted for depressive symptoms in index pregnancy and self-reprted pelvic girdle syndrom, PGS 


\section{Physical activity and postpartum depression in a multiethnic cohort}

Nilam Shakeel , Kåre Rønn Richardsen ${ }^{2}$, Egil W. Martinsen ${ }^{3}$ Malin Eberhard-Gran ${ }^{4,5,6}$ Kari Slinning , Anne Karen Jenum

18

General Practice Research Unit (AFE), Department of General Practice, Institute of Health and Society, University of Oslo

2 Department of Physiotherapy, Faculty of Health Sciences, OsloMet - Oslo Metropolitan

University

3

Division of Mental Health and Addiction, Oslo University Hospital, Institute of

Clinical Medicine, University of Oslo

4, Domain for Mental and Physical Health, Norwegian Institute of Public Health, Oslo, Norway

5

Health Services Research Centre, Akershus University Hospital, Lørenskog

${ }^{6}$ Institute of Clinical Medicine, Campus Ahus, University of Oslo, Norway

${ }^{7}$ Center for Child and Adolescent Mental Health Eastern and Southern Norway (R.BUP Oslo)

Corresonding author:

NS: nilam.shakeel@medisin.uio.no

\section{Co-authors}

KRR : kareri@oslomet.no

EWM: e.w.martinsen@medisin.uio.no

MEG: malin.eberhard-gran@fhi.no

KS: kari.slinning@icloud.com

AKJ: a.k.jenum@medisin.uio.no 


\section{Acknowledgements}

First, the authors thank all the study participants. We also thank study staff at the CHCs in Stovner, Grorud and Bjerke districts in Oslo for collecting the data. Translation of information material and questionnaires was performed by the City Services Department, The Interpreting and Translating Service in Oslo. A special thank to statistician Ragnhild Sørum Falck who helped/assisted us with statistical analysis.

\section{Authors' contributions}

NS and KRRperformed the statistical analysis (except the PCA analyses). NS wrote the first draft-KRRmade the figureand did the multiple imputation analysis. KRRalso helped revising the first draft of the paper. LS contributed with the data acquisition and performed the PCA analyses on socio-economic and integration variables, ME-G, KS, and EWM contributed with expert knowledge about the EPDS and other instruments to capture depression. AKJ initiated and was the project leader of the STORK Groruddalen study. All authors contributed to the interpretation of data, revised the manuscript critically, checked for clarity and content, and approved the final version.

\section{Funding}

The STORK Groruddalen research program was primarily funded by the Norwegian Research Council, with additional funding from The South-Eastern Norway Regional Health Authority, Norwegian Directorate of Health and collaborative partners in The City of Oslo, Stovner, Grorud and Bjerke administrative districts. Nilam Shakeel received funding for a $\mathrm{PhD}$ grant through the Norwegian Research Fund for General Practice. The funder had no involvement in any aspects of the study. 